\title{
Insects on Plants: Explaining the Paradox of Low Diversity within Specialist Herbivore Guilds
}

\author{
Vojtech Novotny, ${ }^{1, \star}$ Scott E. Miller, Jan Hrcek, ${ }^{2}$ Leontine Baje, ${ }^{3}$ Yves Basset, ${ }^{4}$ Owen T. Lewis, ${ }^{5}$ \\ Alan J. A. Stewart, ${ }^{6}$ and George D. Weiblen ${ }^{7}$ \\ 1. Biology Center, Czech Academy of Sciences and Faculty of Science, University of South Bohemia, Branisovska 31, 37005 Ceske \\ Budejovice, Czech Republic; 2. National Museum of Natural History, Smithsonian Institution, Washington, DC 20008; 3. New Guinea \\ Binatang Research Center, P.O. Box 604, Madang, Papua New Guinea; 4. Smithsonian Tropical Research Institute, Apartado 0843- \\ 03092, Balboa, Ancon, Panama; 5. Department of Zoology, University of Oxford, South Parks Road, Oxford OX1 3PS, United \\ Kingdom; 6. School of Life Sciences, University of Sussex, Brighton BN1 9QG, United Kingdom; 7. Department of Plant Biology, \\ University of Minnesota, Saint Paul, Minnesota 55108 \\ Submitted July 24, 2011; Accepted November 7, 2011; Electronically published January 25, 2012 \\ Online enhancement: appendix. Dryad data: http://dx.doi.org/10.5061/dryad.rg155q32.
}

ABstract: Classical niche theory explains the coexistence of species through their exploitation of different resources. Assemblages of herbivores coexisting on a particular plant species are thus expected to be dominated by species from host-specific guilds with narrow, coexistence-facilitating niches rather than by species from generalist guilds. Exactly the opposite pattern is observed for folivores feeding on trees in New Guinea. The least specialized mobile chewers were the most species rich, followed by the moderately specialized semiconcealed and exposed chewers. The highly specialized miners and mesophyll suckers were the least species-rich guilds. The Poisson distribution of herbivore species richness among plant species in specialized guilds and the absence of a negative correlation between species richness in different guilds on the same plant species suggest that these guilds are not saturated with species. We show that herbivore assemblages are enriched with generalists because these are more completely sampled from regional species pools. Herbivore diversity increases as a power function of plant diversity, and the rate of increase is inversely related to host specificity. The relative species diversity among guilds is thus scale dependent, as the importance of specialized guilds increases with plant diversity. Specialized insect guilds may therefore comprise a larger component of overall diversity in the tropics (where they are also poorly known taxonomically) than in the temperate zone, which has lower plant diversity.

Keywords: rainforest, New Guinea, host specialization, niche theory, community saturation, species pool.

\section{Introduction}

Host specialization of insects plays a central role in theories that explain the extraordinary diversity of plant-insect food webs in tropical forests. High plant diversity can be pro-

\footnotetext{
* Author for correspondence; e-mail: novotny@entu.cas.cz.
}

Am. Nat. 2012. Vol. 179, pp. 351-362. (c) 2012 by The University of Chicago. 0003-0147/2012/17903-53210\$15.00. All rights reserved. DOI: $10.1086 / 664082$ moted where specialized herbivores or pathogens have density-dependent effects on plant growth and fitness, putting locally rare plant species at an advantage (the JanzenConnell hypothesis, reviewed in Leigh et al. 2004). High herbivore diversity can be promoted if plant-herbivore interactions are specialized, because finely partitioned plant resources will facilitate species coexistence (Chase and Leibold 2003; Lewinsohn and Roslin 2008).

Insect herbivore species feeding on only one or a few plant species are often also highly specialized in their use of resources on a particular plant, while those species feeding on a wide range of plant species tend to also have a more generalized use of resources within each plant species. For instance, many generalist adult chewers feed opportunistically on both the flowers and the leaves of their numerous host species (Kishimoto-Yamada and Itioka 2008), while the more host-specific species often use only particular plant parts. Some fruit fly species use only certain parts of either male or female flowers (Condon et al. 2008), and many miners use only a specific part of only one side of the leaf (Hespenheide 1991) from their only host species. Generalist species also tend to have more plasticity in response to environmental conditions (Nylin and Janz 2009). The positive correlation between herbivore specialization to plant species and to plant resources within a particular plant species implies that the assemblages of herbivorous species coexisting on a particular plant species should be dominated by species from host-specific guilds of herbivores with narrow, coexistence-facilitating niches, rather than by species from generalist guilds.

The prominence of specialists in herbivore assemblages would be further increased if they used resources more efficiently than generalists, so that the same amount of resources could support more individuals, and therefore 
species, of specialists than generalists. Such a trade-off between feeding efficiency and host plant specialization has been suggested, but the evidence remains equivocal (Jaenike 1990; Joshi and Thompson 1995).

Since the classical study of bracken fern herbivores (Lawton et al. 1993) was published, it has become increasingly clear that insect herbivore communities are rarely saturated with species and their diversity is determined not only by the defense traits of plants (Agrawal 2007) but also by the size of the regional species pool from which they are assembled (Caley and Schluter 1997; Ricklefs 2004; Harrison and Cornell 2008). Their taxonomic composition mirrors the composition of the regional species pool (Frenzel and Brandl 2000). More specialized herbivore guilds could thus be relatively species poor as a consequence of smaller regional pools of species available to these guilds and/or a lower proportion of these species pools being included in local assemblages compared with the more generalist guilds.

While herbivorous lineages speciate more rapidly than nonherbivorous ones (Mitter et al. 1988; Farrell 1998), the effect of host specialization on the size of regional pools of herbivorous species remains poorly known, particularly since the relationship between host range and speciation rate is complex and variable among herbivorous lineages (Nosil 2002; Janz and Nylin 2008). If the regional species pools of generalist and specialist guilds were of equal size, each plant species would have more generalist species than specialists that were able to feed on it regionally. The proportional sampling of these species pools by local assemblages could thus produce a positive correlation between host range and species richness in assemblages of herbivores.

In this study we use folivorous insects to explore the determinants of species diversity in herbivorous assemblages. Folivores use a well-defined resource, the leaf, in a variety of ways, including various combinations of exposed, semiconcealed, and endophytic chewing or sucking by larvae or adults. Folivores thus form five well-defined guilds feeding on the same plant tissue, making them well suited for our study of the relationship between species richness, abundance, and host specificity. We perform these analyses on highly diverse folivorous assemblages feeding on 38 species of woody plants in a lowland tropical rainforest in New Guinea. Our main goal is to explain why, in folivorous herbivores at least, we observe exactly the opposite pattern to that expected from classical niche theory: the least specialized guild of mobile chewers has the highest number of species, while highly specialized miners and mesophyll suckers are species poor (Novotny et al. 2010).

\section{Methods \\ Insect Sampling and Taxonomic Analysis}

We define folivores as herbivores feeding on leaf mesophyll tissue, thus excluding the sucking species that tap phloem or xylem vessels as well as gallers, which feed on specific tissue that they induce. Folivores were classified into guilds according to their feeding mode (chewing, sucking) and the intimacy of the association with their host plant (modified from Novotny et al. 2010):

Mobile chewers: All externally feeding hemimetabolous larvae and all adults (Orthoptera, Phasmatodea, and Coleoptera), dominated in our samples by Chrysomelidae, Cerambycidae, Curculionidae, and Tettigoniidae.

Exposed chewers: All externally feeding and fully exposed holometabolous larvae (Lepidoptera, Coleoptera), dominated by Geometridae, Noctuidae, and Lymantriidae.

Semiconcealed chewers: All leaf rolling and leaf tying holometabolous larvae (Lepidoptera), dominated by Crambidae, Tortricidae, and Choreutidae.

Miners: All leaf mining species (Lepidoptera, Coleoptera, Diptera), dominated by Gracillariidae.

Mesophyll suckers: This guild includes two exposed taxa with distinct feeding modes, the cicadellid subfamily of Typhlocybinae (Auchenorrhyncha), which sucks individual mesophyll cells, and species of Heteroptera, which dissolves and sucks leaf tissues (Andrew and Hughes 2005). Only the former group was studied here.

While the internal and semiconcealed external larvae are restricted to an individual plant, the exposed holometabolous larvae have a limited ability to move among plant individuals and species, and hemimetabolous larvae and adults are typically mobile, able to repeatedly change host species and individuals during their lifetimes. Further, holometabolous larvae and adults of the same species often feed on different plant species and exhibit different levels of host specificity (Pokon et al. 2005) and should therefore be analyzed separately.

Within each of the guilds defined here, the full range of herbivorous taxa was investigated with the exception of the mesophyll suckers, which can be considered a taxon guild (sensu Simberloff and Dayan 1991), since our study is limited to Auchenorrhyncha. Folivorous guilds were sampled from 38 plant species at three study sites (Baitabag, Ohu, and Mis Villages) located within a $10 \times 20-$ $\mathrm{km}$ area encompassing a mosaic of secondary and primary lowland hill forest in Madang Province (Papua New Guinea) for at least 1 year between 1995 and 2008. Plant selection included all major plant lineages (14 families and 23 genera) as well as three clusters of closely related, congeneric species (11 Ficus, 4 Macaranga, and 3 Psychotria species). The studied plant species are listed in table Al, 
available online, and their phylogenetic relationships are shown in figure A1, also available online.

Each plant species was sampled with equal effort for each guild, removing potential effects due to variations in host plant abundance. The foliage area sampled amounted to $1,500 \mathrm{~m}^{2}$ per species for all plant species and guilds. The insects were handcollected from foliage and brought to the laboratory. Adults were tested for feeding on the plant species from which they were collected; only insects that fed were included in the analyses. Larvae were provided with fresh leaves of the plant species from which they were collected and were reared in containers or plastic bags. Twigs with leaves including miners were placed in plastic bags, and the insects were reared to adults (see Novotny et al. 2002, 2010 for details).

Larvae from all guilds except mesophyll suckers and all adults were assigned to species. All adult insects were later identified by specialists as far as possible (Miller et al. 2003), and larval species were linked to corresponding adult species. Species identifications were often verified by dissection of genitalia and reference to type specimens. For Typhlocybinae, only males were used in the taxonomic analysis, as the taxonomy of this group is based mostly on the morphology of the male genitalia whereas females are often impossible to identify morphologically. Numbers of Typhlocybinae were multiplied by two to account for missing females. Comparisons of mitochondrial cytochrome oxidase I DNA sequence divergence with morphology were used for $\sim 10,000$ insect specimens (Lepidoptera in all guilds and Coleoptera and Diptera in miners) to verify our species boundaries, including polymorphic species and cases of sexual dimorphism (Hulcr et al. 2007a; Craft et al. 2010).

Plant vouchers are deposited in the Papua New Guinea Forest Research Institute in Lae and the University of Minnesota in St. Paul, and insect vouchers are deposited in the Bishop Museum in Honolulu, the Smithsonian Institution in Washington, DC, the Papua New Guinea Agriculture Research Institute in Port Moresby, and the Natural History Museum in London. Lepidopteran species are detailed at http://www.entu.cas.cz/png/caterpillars. The raw data on the composition of herbivorous guilds are deposited in the Dryad repository (Dryad data: http:// dx.doi.org/10.5061/dryad.rg155q32).

\section{Food Web Analysis}

The food web between $i=1, \ldots, H$ folivorous species and $j=1, \ldots, P$ plant species was defined by the number of herbivore individuals $i$ feeding on plant $j\left(n_{i j}\right)$ for each trophic interaction. Trophic relationships supported by only a single feeding individual were excluded as poorly documented. Each folivorous assemblage on a particular
Table 1: Herbivore-plant matrix notation and food web parameters

\begin{tabular}{lccccc}
\hline & \multicolumn{5}{c}{ Plant species } \\
\cline { 2 - 6 } Herbivore species & 1 & $j$ & $P$ & $\Sigma$ & Count \\
\hline 1 & $n_{11}$ & & $n_{1 p}$ & $n_{1}$ & $p_{1}$ \\
$i$ & & $n_{i j}$ & & $n_{i}$ & $p_{i}$ \\
$H$ & $n_{h 1}$ & & $n_{h p}$ & $n_{h \cdot}$ & $p_{h}$ \\
$\Sigma$ & $n_{\cdot 1}$ & $n_{\cdot j}$ & $n_{\cdot p}$ & $n .$. & \\
Count & $h_{1}$ & $h_{j}$ & $h_{p}$ & & \\
\hline
\end{tabular}

Note: $i=$ herbivore species $1, \ldots, i, \ldots, H ; j=$ plant species $1, \ldots$, $j, \ldots, P ; n_{i j}=$ the number of individuals of herbivore $i$ feeding on plant $j ; n_{. j}=$ herbivore load, that is, the number of herbivores on plant species $j$ (average: $\bar{n}_{. j}=n . / P$ ); $n_{i}$. = population size, that is, the number of herbivores from species $i$ (average: $\bar{n}_{i \cdot}=n . / H$ ); $h_{j}=$ species richness, that is, the number of herbivore species on plant species $j$ (average: $\bar{h}_{j}=\sum h_{j} / P$; note that $\sum h_{j}=\sum p_{i}$ ); $p_{i}=$ host plant range, that is, the number of host plant species of herbivorous species $i$ (average: $\bar{p}_{i}=\sum p_{i} / H$; average host range on plant species $j: \bar{p}_{i j}=$ $\left.\sum_{n_{i j} \neq 0} p_{i} / h_{j}\right) ; 1 / p_{i}=$ herbivore host specificity. Note that $\bar{h}_{j}$ can be expressed in terms of $\bar{p}_{i}, \bar{n}_{. j}$, and $\bar{n}_{i}$. as follows: $\bar{p}_{i} \times \bar{n}_{. j} / \bar{n}_{i}=$ $\left(\sum p_{i} / H\right) \times(n \ldots / P) /(n \ldots / H)=\sum p_{i} / P=\sum h_{j} / P=\bar{h}_{j}$.

plant species was characterized by species richness $h_{j}$ (the number of herbivore species on plant species $j$ ) and herbivore load $n_{. j}$ (the number of herbivore individuals on plant species $j$ ), while each herbivore species was characterized by host range $p_{i}$ (the number of host plant species of herbivorous species $i$ ) and population size $n_{i}$. (the number of herbivore individuals from species $i$ ). Folivorous guilds were characterized by average values of these parameters (table 1).

The herbivorous species and guilds are not classified here as specialists or generalists, as such classifications can be misleading (Novotny and Basset 2005). Instead, we measure their host specificity quantitatively, using $p_{i}$ for species and $\bar{p}_{i}$ for guilds, and we use the terms "specialist" and "generalist" only in reference to the relatively low or high values of these parameters, respectively.

The number of herbivore species $\left(H_{500}\right)$ and trophic interactions $\left(T_{500}\right)$ expected in a random subsample of 500 herbivore individuals was determined by rarefaction respectively applied to $n_{i}$. and $n_{i j}$ values in the plant-herbivore matrix for each guild. The rarefied host range $p_{500}$ was estimated as $T_{500} / H_{500}$.

The interguild correlations across the 38 plant species were calculated for species richness $\left(h_{j}\right)$ and herbivore load $\left(n_{. j}\right)$ as Pearson $r$ on independent contrasts, using cladogram topology and branch lengths from the molecular phylogeny constructed for the studied plant species (fig. A1), using Compare 4.6. software (Martins 2003).

The accumulation of herbivore species with increasing number of plant species was described by the Mao Tau function, an analytical analog of a randomized speciesaccumulation curve, implemented in the program 
EstimateS (Colwell 2008). The trends in herbivore species richness were extrapolated to 10,000 plant species (the approximate number of woody plants known from New Guinea; Hoft 1992), using power functions fitted to the species-accumulation curve for 10-38 plant species.

The regional species pool was defined for the purposes of this study as the set of lowland rainforest species within a zone of approximately $10,000 \mathrm{~km}^{2}$ surrounding the study area, a definition that thus combines both spatial and ecological criteria. The regional species pool for the exposed and semiconcealed chewers was estimated using samples from an approximately equidistant grid of eight sites within a $500 \times 150-\mathrm{km}$ area of lowland rainforest, including our present study area. The caterpillars were sampled from 15 plant species at each site, using the same methods and sample size as this study. This data set was further expanded using caterpillar samples from 50 plant species sampled at our present study sites in addition to the 38 plant species already included in this study. The combined data set thus included herbivores feeding on a
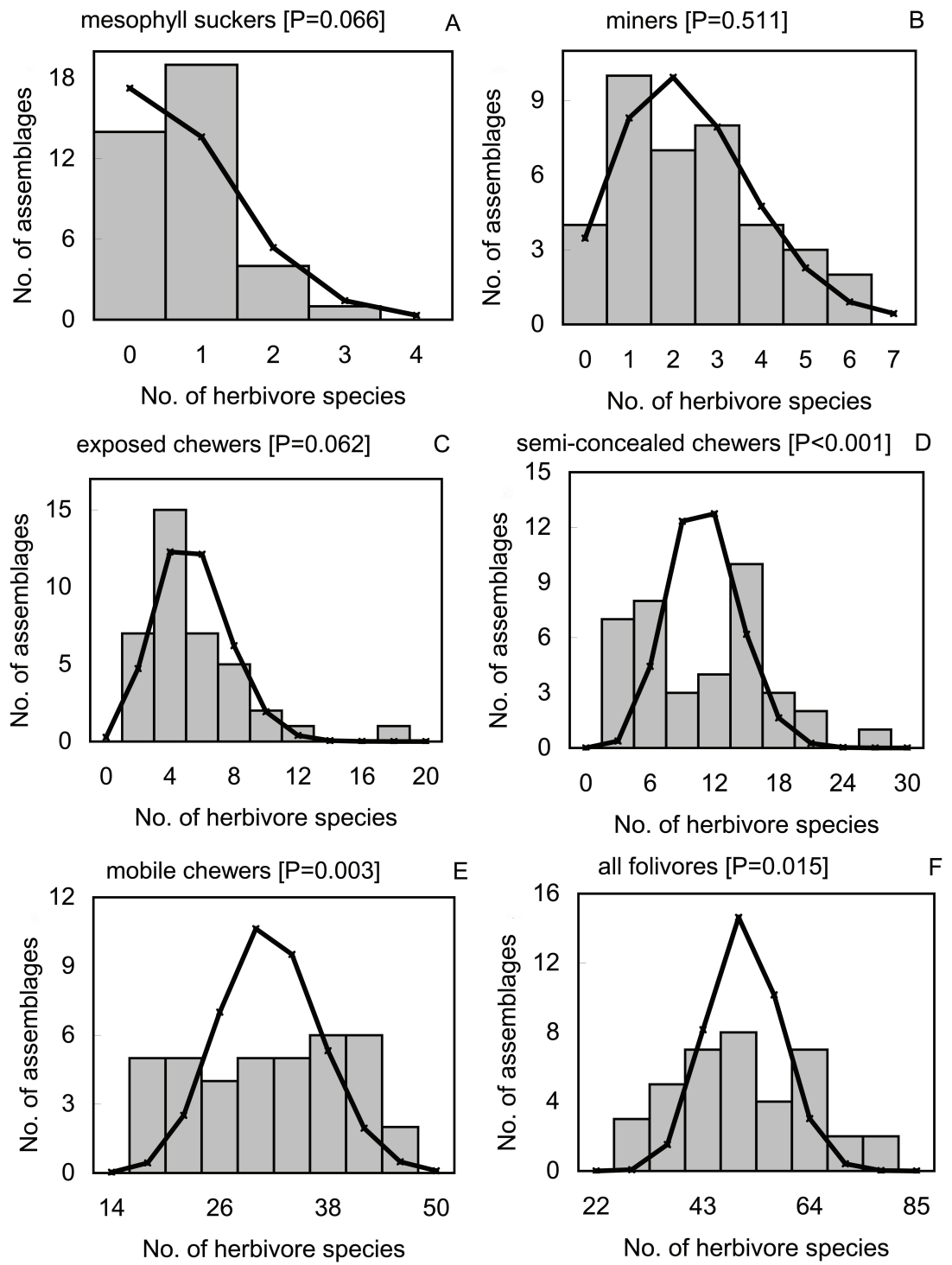

Figure 1: Distribution of species-richness values (number of species on individual plant species, $h_{j}$ ) observed in different folivorous guilds $(A-E)$ and the entire folivorous assemblage $(F)$, with expected values for a Poisson distribution (line). The $h_{j}$ distribution is significantly different from a Poisson distribution in semiconcealed chewers $(D)$, mobile chewers $(E)$, and all folivores $\left(F ; \chi^{2}\right.$ tests, $P$ value is reported above each histogram). 
Table 2: Between-guild correlations of herbivore species richness $\left(h_{j}\right.$; lower half of the matrix) and herbivore load $\left(n_{. j}\right.$; upper half of the matrix) for the 38 plant species studied

\begin{tabular}{lccccc}
\hline & $\begin{array}{c}\text { Mobile } \\
\text { chewers }\end{array}$ & $\begin{array}{c}\text { Exposed } \\
\text { chewers }\end{array}$ & $\begin{array}{c}\text { Semiconcealed } \\
\text { chewers }\end{array}$ & Miners & $\begin{array}{c}\text { Mesophyll } \\
\text { suckers }\end{array}$ \\
\hline Mobile chewers & & -.325 &. $\mathbf{3 4 4}$ & .082 & $\underline{.464}$ \\
Exposed chewers & .275 & & -.355 & .024 & .108 \\
Semiconcealed chewers & .234 & .044 & & .276 & -.206 \\
Miners & .286 & .171 & -.317 & & -.074 \\
Mesophyll suckers &. $\mathbf{3 7 2}$ & .135 & -.264 & .313 & \\
\hline
\end{tabular}

Note: Pearson correlation on independent contrasts based on the phylogeny of the plant species (fig. A1, available online), $n=37$. Correlations with $P<.05$ are in bold; the correlation with $P<.005$ (significant after Bonferroni correction for 10 analyses) is underlined.

wide array of plant species (94 species from 32 families) in a particular habitat, lowland rainforest, from eight sites distributed over a large geographic area. This regional data set included 91,544 individuals and 524 species of semiconcealed chewers and 32,342 individuals and 538 species of exposed chewers (details in Novotny et al. 2007). After excluding plant-insect interactions supported by only a single feeding individual, the regional pools of 258 species of exposed herbivores and 268 species of semiconcealed herbivores were used in the analysis.

\section{Results}

We documented feeding interactions for 49,597 herbivore individuals of 865 species involved in 3,260 trophic interactions with the 38 species of plants studied. This data set was reduced to 48,170 herbivore individuals from 545 species and 1,833 trophic interactions by excluding 1,427 trophic interactions supported by single herbivorous individuals.

The distribution of herbivore species-richness $\left(h_{j}\right)$ values among plant species was not significantly different from a Poisson distribution in the three low-diversity guilds (mesophyll suckers, miners, and exposed chewers), while the species-rich guilds of semiconcealed and mobile chewers and the entire assemblage of folivores exhibited significantly higher variance in $h_{j}$ than predicted by a Poisson distribution (fig. 1).

Herbivore species richness $\left(h_{j}\right)$ varied among plant species idiosyncratically in each guild, largely independently of other guilds. The independent contrasts correlation between the $h_{j}$ values of different guilds across the 38 studied plant species was statistically significant for only one of the 10 possible pairs formed by the five guilds: mobile chewers versus mesophyll suckers. Likewise, the herbivore load $\left(n_{\cdot j}\right)$ did not show any consistent correlation between guilds across plant species (table 2).

Folivorous guilds varied widely in their species richness $\left(\bar{h}_{j}=0.8-30.1\right.$ species per plant species), host range $\left(\bar{p}_{i}=1.2-5.2\right.$ host species per herbivore species), and her- bivore load on plant species $\left(\bar{n}_{\cdot j}=17-507\right.$ individuals per $1,500 \mathrm{~m}^{2}$ of leaf foliage; table 3 ). These three parameters were all positively correlated among folivorous guilds (fig. 2 ), while none of them was correlated with herbivore population size $\bar{n}_{i}$. (Pearson $r, P>$.05). In stepwise multiple regression of $\bar{p}_{i}, \bar{n}_{i, j}$ and $\bar{n}_{i}$. on $\bar{h}_{i}$, only host range $\bar{p}_{i}$ remained a significant predictor of species richness $(r=$ $0.984, P=.003$; fig. $2 A$ ). Folivorous guilds could thus be ordered from the species-rich and generalist guild of mobile chewers through the intermediate guilds of semiconcealed and exposed chewers to the species-poor and hostspecific guilds of miners and mesophyll suckers (fig. 3). Herbivore load was predicted by host range as the sole variable in multiple regression of $\bar{h}_{j}$, $\bar{p}_{i}$, and $\bar{n}_{i \cdot}$. on $\bar{n}_{\cdot j}$ $(r=0.815, P=.007$; fig. $2 B)$.

The total species richness of folivorous guilds on all 38 plant species $(H)$ was correlated with average host range $\bar{p}_{i}$ (fig. $2 C$ ). This correlation persisted when the herbivore abundance in each guild was standardized to 500 individuals by rarefaction. The rarefied species richness $H_{500}$, ranging from 25 species in mesophyll suckers to 80 species in mobile chewers, and the host range $p_{500}$, ranging from 1.2 plant species in mesophyll suckers to 3.3 plant species in mobile chewers, were correlated across folivorous guilds (Spearman's $r=1.00, P=.017, n=5$; table 3 ).

The distribution of species richness among plant species in individual guilds, reported in figure 1, as well as the relationships between the principal food web parameters $\bar{h}_{j}, \bar{p}_{i}, \bar{n}_{. j}$, and $H$, reported in figure 2 , were also recalculated using a complete, unfiltered data set including singleton trophic interactions, with similar results (figs. A2, A3, available online). The data filtering reduced the average herbivore species richness $\bar{h}_{j}$ to $56 \%$ of the original value and the average herbivore host range $\bar{p}_{i}$ to $90 \%$ of the original value for all folivorous herbivores (table 3 ).

The accumulation of species richness $H$ with increasing number of plant species $P$ progressed at a different rate in each guild, depending on its host specificity. The herbivore species accumulation from 10 to 38 species was accurately described by the power function $H=k \times P^{z}$, 
Table 3: Food web statistics for folivorous guilds and the entire folivorous assemblage

\begin{tabular}{|c|c|c|c|c|c|c|c|c|}
\hline Guild & $\bar{h}_{j}$ & $\bar{p}_{i}$ & $\bar{n}_{\cdot j}$ & $\bar{n}_{i}$ & $H$ & $H_{500}$ & $T_{500}$ & $p_{500}$ \\
\hline Mobile chewers & $30.1(1.39)$ & $5.2(.48)$ & $507.2(36.19)$ & $87.2(19.75)$ & 221 & 79.5 & 261.7 & 3.3 \\
\hline Semiconcealed che & $10.1(1.03)$ & $3.1(.31)$ & $338.6(39.81)$ & $105.5(19.07)$ & 122 & 71.9 & 134.3 & 1.9 \\
\hline Exposed chewers & $4.9(.51)$ & $1.7(.15)$ & $151.7(37.70)$ & $53.4(9.27)$ & 108 & 66.6 & 88.3 & 1.3 \\
\hline Miners & $2.4(.27)$ & $1.3(.12)$ & $253.3(32.59)$ & $139.5(19.78)$ & 69 & 49.8 & 62.4 & 1.3 \\
\hline Mesophyll suckers & $.8(.12)$ & $1.2(.08)$ & $16.9(3.68)$ & $25.7(5.72)$ & 25 & 25.0 & 30.0 & 1.2 \\
\hline All folivores & $48.2(2.23)$ & $3.4(.22)$ & $1,267.6(76.32)$ & 88.4 ( 9.65$)$ & 545 & 164.0 & 282.8 & 1.7 \\
\hline All folivores (full data set) & $85.8(3.41)$ & $3.8(.19)$ & $1,305.8(76.60)$ & $57.4(6.26)$ & 865 & 172.6 & 291.2 & 1.7 \\
\hline
\end{tabular}

Note: Data are mean (SE). Number of plant species $P=38$ for all guilds. $H_{500}$ and $T_{500}=$ total number of herbivore species and trophic interactions, respectively, in a random subsample of 500 individuals from each guild; $p_{500}=$ average host plant range in a random subsample of 500 individuals from each guild. Full data set also includes trophic interactions supported by single individuals. See table 1 for other notation and table 2 for correlations between the parameters.

where $k$ is the estimated number of herbivore individuals on a single plant species and $z$ is the rate of species accumulation. The $z$ values ranged among guilds from 0.454 in the least specialized mobile chewers to 0.893 in the most specialized mesophyll suckers (fig. 4).

In exposed feeders, the local species richness $\bar{h}_{j}=4.9$ $( \pm 0.51)$ represented $1.9 \%$ of the regional species pool of 258 species. In semiconcealed feeders, $\bar{h}_{j}=10.1( \pm 1.03)$ represented $3.8 \%$ of the regional species pool of 268 species. For the local host range of exposed feeders $\bar{p}_{i}=$ $1.7( \pm 0.15)$ plant species represented $58 \%$ of their regional host range of $2.94( \pm 0.23)$ plant species, while for semiconcealed feeders, the local range of $\bar{p}_{i}=3.1( \pm 0.31)$ host species represented $74 \%$ of their regional range of 4.2 $( \pm 0.38)$ host species.

\section{Discussion}

\section{Weak Trophic Interactions in Plant-Herbivore Food Webs}

Plant and insect communities in tropical rainforests comprise many rare species, and rainforest food webs include many rare trophic interactions. In particular, even very large samples from rainforest food webs include numerous species and trophic interactions documented by single individuals (singletons; Novotny and Basset 2000). While some of these interactions may be important for the dynamics of food webs, others represent marginal or unusual host plant choices or erroneous records. Our analysis was conservative, excluding all singleton species and interactions.

Data filtering had surprisingly little effect on the relationships between the fundamental parameters of plantherbivore food webs $\left(\bar{h}_{j}, \bar{p}_{i}, \bar{n}_{. j}\right.$, and $\left.H\right)$. Predictably, it had a major effect on the estimates of species richness $\left(\bar{h}_{j}\right)$. In contrast, the estimates of host range $\left(\bar{p}_{i}\right)$ changed little, as the filtered data set lacked all singleton species, which can only have one recorded host plant species, and many singleton host records for more generalist species.

\section{Species Richness of Folivores on Individual Host Plants}

The distribution of species richness among plant species conformed to a Poisson distribution, suggesting that colonization events can be seen as mutually independent events; the probability of a plant species acquiring a new herbivore is not influenced by the number of herbivore species already present on that plant species. This is not the case for the two herbivorous guilds with $\geq 5$ herbivore species per plant species, where both low-diversity and high-diversity assemblages are more frequent than expected. Plant species are thus not colonized with uniform probability, as some of them are particularly suitable or unsuitable for the particular herbivorous guild. In contrast, the number of plant species entirely devoid of mesophyll suckers or miners is predicted well by a Poisson distribution for these species-poor guilds and thus does not necessarily require any specific explanation based on plant life-history traits.

Species richness and herbivore load in different folivorous guilds feeding on the same plant species may not be mutually independent. They can be correlated either negatively (because species from one guild can competitively exclude species from another guild) or positively (because certain functional traits of the plant species, such as its antiherbivore defenses, have similar impacts on species from more than one guild). Across plant species, competitive interactions are expected to lead to a negative correlation between species richness (and herbivore load) in different guilds, while a shared impact of the host plant species' traits leads to a positive correlation. In folivorous herbivores, both species richness and herbivore load varied largely independently among guilds. This result confirmed our expectations that interguild competition is not strong enough to generate a negative correlation between species richness in different guilds. It is also consistent with the lack of such correlations observed for insects feeding on temperate zone trees (Claridge and Wilson 1981; Cornell and Kahn 1989; Kelly and Southwood 1999). 
A

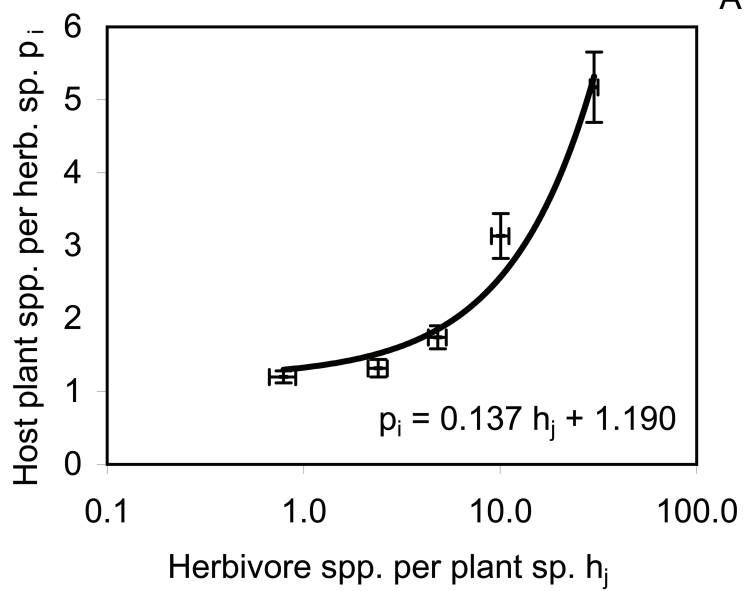

B

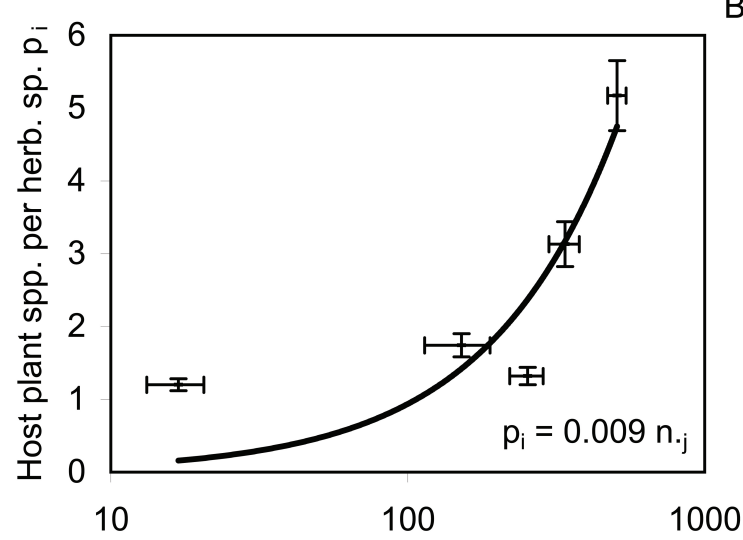

Herbivore ind. per plant sp. $\mathrm{n}_{\cdot \mathrm{j}}$

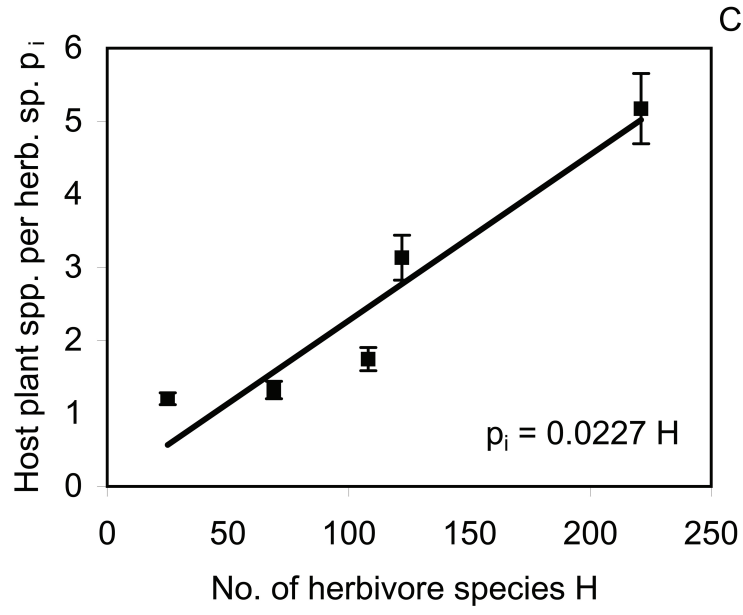

Figure 2: Relationships between average herbivore host range $\bar{p}_{i}$ and species richness $\bar{h}_{j}(A ; r=0.984, P=.003)$, herbivore load $\bar{n}_{. j}(B$; $r=0.897, P=.039)$, and total herbivore species richness $H(C$; $r=0.954, P=.012)$. Species richness $\bar{h}_{j}$ is also correlated with herbivore load $\bar{n}_{. j}(r=0.879, P=.05)$. See table 1 for definitions of the variables.
Species Richness, Host Range, and Herbivore Load

The species richness of herbivores $\left(\bar{h}_{j}\right)$ can be expressed in terms of host range $\left(\bar{p}_{i}\right)$, herbivore load on plant species $\left(\bar{n}_{. j}\right)$, and herbivore population size $\left(\bar{n}_{i}\right.$; table 1$)$ :

$$
\bar{h}_{j}=\bar{p}_{i} \times \bar{n}_{\cdot j} / \bar{n}_{i} \cdot .
$$

The ability of plant species to support large numbers of herbivores $\left(\bar{n}_{. j}\right)$ and the ability of herbivores to survive in small populations $\left(\bar{n}_{i}\right.$. $)$ should indeed lead to high species richness of herbivores in each guild $(H)$. Their host range $\left(\bar{p}_{i}\right)$ then determines the species richness on individual plant species $\left(\bar{h}_{j}\right)$, as generalist species contribute to the species richness of a larger number of plant species than specialist species do. Our analysis suggests that of these three parameters, host range $\bar{p}_{i}$ best explains species richness $h_{j}$ across the folivorous guilds, and it is also the best predictor of herbivore load on plant species $\bar{n}_{. j}$.

The species richness of herbivores can also be expressed in terms of host range and the total number of herbivore $(H)$ and plant $(P)$ species (table 1 ):

$$
\bar{h}_{j}=H \times \bar{p}_{i} / P .
$$

This relationship can be used to predict the total number of herbivore species $H$ from their host range $\bar{p}_{i}$, assuming that there is no correlation between host range and $\bar{h}_{j}$; that is, that a particular plant species can support the same number of species from specialized as well as generalist guilds of herbivores. We used the average value of herbivore species richness for all five folivorous guilds $\bar{h}_{j}=9.66$ and predicted the total number of herbivores as $H=\bar{h}_{j} \times P / \bar{p}_{i}$. This simple null model generated a negative correlation between $H$ and $\bar{p}_{i}$, since the most specialized guilds were also predicted to be the most rich in species (fig. 5).

In contrast, our data revealed a positive correlation between $\mathrm{H}$ and $\bar{p}_{i}$. This is at variance with the prediction from niche theory that specialist species will have narrower niches, facilitating coexistence in herbivore assemblages. The observed number of herbivore species in each guild is thus more likely determined by the diversity of their regional species pools. Narrow host range also appears to be correlated with low species richness in nonfolivorous guilds: fruit flies (Novotny et al. 2005), gallers (P. Butterill, unpublished data), and bark beetles feeding on phloem tissue (Hulcr et al. 2007b) all had $\bar{h}_{j} \leq 3.0$ on tropical trees in our study area. Generalist guilds, on the other hand, exhibit a wide range of species-richness values, from species-rich mobile chewers to species-poor xylem suckers (Novotny et al. 2010).

Our analysis is limited by the sampling universe, which comprised only five guilds and 38 plant species. The accuracy of each parameter could be improved by sampling 
more plant species for each guild, but increasing the total number of data points would require a narrower definition of each guild (such as dividing semiconcealed chewers into leaf tiers and leaf rollers). The analysis of each phylogenetically independent entry into each guild may be a more useful approach in future analyses. Guild membership is often evolutionarily very flexible (Nyman et al. 2006), so that most of the guilds comprise numerous insect lineages sharing their feeding mode and resource base as a result of convergent evolution. The only exception for the folivores studied here is the guild of mesophyll suckers, for which the feeding mode of piercing and emptying individual mesophyll cells might have evolved only once from phloem feeding (Novotny and Wilson 1997).

Many folivorous species consume resources other than leaves during part of their life cycle. Thus, their distribution and abundance may be influenced by the availability of other resources. This may be the case for the larvae of 164 beetle species (mostly Chrysomelidae, Cerambycidae, and Curculionidae), with mostly generalist adults in the mobile-chewers guild. However, our studies suggest that these larvae, which usually feed on plant parts other than leaves, are also mostly generalists. The larvae of only one of these species, Promechus bimaculatus, were sampled from leaves, while we reared 44 species from roots (Pokon et al. 2005), 20 species from wood (Novotny et al. 2010), and one species from fruits (R. Ctvrtecka, unpublished data). Although we do not have data for the remaining 98 species, it is clear that most of the Chry- somelidae species that fed on leaves as adults had generalist larvae that fed on roots (Pokon et al. 2005), and the Cerambycidae species had wood-boring larvae that were also mostly generalist (Novotny et al. 2010). There are only some Curculionidae species, particularly those with larvae that feed on seeds, for which larvae could be more specialized than their folivorous adults.

\section{Species Richness in Assemblages and Regional Species Pools}

Regional species pools are not sufficiently known for any folivorous guilds in New Guinea or elsewhere in the tropics (Novotny and Missa 2000). Our approximation of regional species pools for exposed and semiconcealed feeders is far from complete, since even at our best-studied site we were able to document only $<20 \%$ of the estimated plant-herbivore trophic interactions (Novotny et al. 2010). Our regional-pool estimate may be even less complete. We therefore compared the ratios of local to regional species richness and local versus regional differences in host specificity between two folivorous guilds, using identical local and regional sample sizes for both guilds. The standardized sample size permitted comparison between the two guilds, while the absolute values of the local to regional speciesrichness ratios were likely biased in each guild by incomplete sampling.

In regional pools of $H_{r}$ herbivore species and $P_{r}$ plant species, a single plant species in a local community could

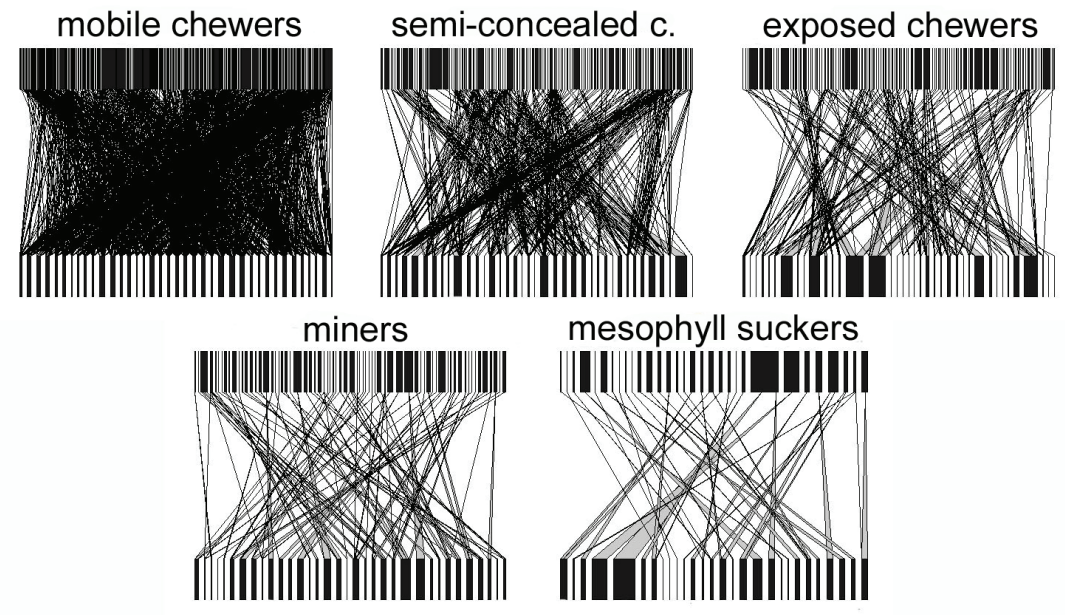

Figure 3: Quantitative plant-herbivore food webs for folivorous guilds feeding on 38 plant species. For each web, the lower bars represent the frequency with which each host plant is consumed by herbivores and the upper bars represent herbivore abundance. The widths of the links between trophic levels are proportional to the frequency of each interaction. Herbivores from each guild were sampled with equal effort on all plant species. Plant and herbivore species included in the food webs are listed in table A1, available online, and the raw data on the guild composition are deposited in the Dryad repository (Dryad data: http://dx.doi.org/10.5061/dryad.rg155q32). 
be used by $\bar{h}_{j}=H_{r} \times \bar{p}_{i} / P_{r}$ species of herbivores. A positive correlation between local species richness and host range of herbivores is thus expected for communities that are random subsamples from regional species pools of equal size for each guild.

Our results suggest that local communities sample regional species pools nonrandomly, in a way that further strengthens the positive correlation between herbivore species richness and their host range in communities. In our two-guild regional species pool, $49 \%$ of species were exposed feeders and $51 \%$ of species were semiconcealed feeders. Random sampling, where every plant-herbivore trophic interaction in this pool had the same probability of being represented locally, would reduce the share of exposed feeders to $40 \%$ of species, reflecting their smaller host ranges relative to those of the semiconcealed feeders. However, we found that exposed feeders colonized a lower proportion of regionally documented host plant species in local communities than the semiconcealed feeders. This reduced their share to $32 \%$ of species in local communities. The number of species in less specialized guilds, such as semiconcealed feeders, is thus elevated by more intense sampling from regional species pools compared with the specialized guilds. This colonization bias has also been noted in other plant-herbivore food webs (Fox and Morrow 1981). It might arise from a higher risk of local extinction in more specialized herbivores, particularly those feeding on host species occurring at low densities.

Host range may also drive the size of the regional species pool by affecting speciation rates. Restricted gene flow among conspecific populations feeding on different host plant species could increase rates of speciation in generalist taxa as, in the extreme, each host-associated population could behave as a distinct species (Dres and Mallet 2002; Nosil et al. 2002). Our analysis of molecular variance revealed host-associated differences in the populations of $35 \%$ of species from exposed and semiconcealed guilds of chewers in our study system (Craft et al. 2010). The relationship between host range and speciation rate is, however, complex and variable among herbivore lineages (Nosil 2002; Janz and Nylin 2008).

While niches in herbivorous guilds may remain vacant (Lawton et al. 1993), some generalist species feeding on a particular plant species may not be able to maintain a viable population on that plant species alone. For instance, the optimum diet of several coexisting generalist grasshopper species included the same host plant species, but in different proportions (Behmer and Joern 2008). The species richness of generalist herbivores on individual plant species may thus be inflated by species that require additional host plant species in order to sustain their populations. This species enrichment in generalist guilds would be an example of the mass effect observed in many communities (Shmida and
Wilson 1985; Leibold et al. 2004). We can consider such communities on individual plant species to be "supersaturated," as they would lose some of their generalist herbivore species if they were isolated from the neighboring communities feeding on other plant species.

The relative diversity of the regional species pools among guilds depends entirely on the spatial scale that is considered to be "regional." Species richness of herbivores $(H)$ accumulates with plant diversity $(P)$ following a power function $H=k \times P^{z}$, where $z$ varies with host specificity from 0 when all herbivore species feed on all plant species to 1 when all herbivore species are strictly monophagous. The observed $z$ values ranged from 0.454 in the generalist mobile chewers to 0.893 in the specialized mesophyll suckers. The generalist guilds thus have the highest species richness in local assemblages and the lowest rate of species accumulation with plant species diversity. The relative species richness of guilds is dependent on plant diversity. For example, the mobile-chewers guild is the most species rich on a single plant species but is predicted to rank only fourth when 10,000 plant species are considered (fig. 4). The extremely long-range extrapolation of herbivore species richness to 10,000 plant species was used only to illustrate trends in relative species diversity among guilds and not to estimate herbivore species richness for large floras. The number of herbivore species is a power function of the number of plant species, which in turn is a power

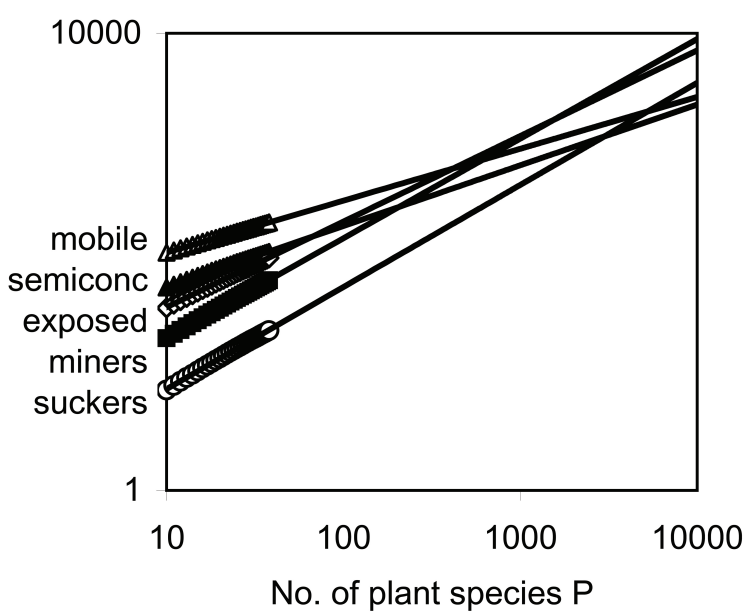

Figure 4: Species accumulation in the five folivorous guilds on 38 plant species. The Mao Tau species-accumulation curves are shown for herbivore species on 10-38 plant species amalgamated in random order and extrapolated to 10,000 plant species, using power functions (mobile chewers: $y=42.48 \times x^{0.454}$; semiconcealed chewers: $y=$ $17.86 \times x^{0.531}$; exposed chewers: $y=7.15 \times x^{0.749}$; miners: $y=$ $2.91 \times x^{0.871}$; mesophyll suckers: $y=0.99 \times x^{0.893} ; n=29, R^{2}>$ 0.999 for all guilds). 


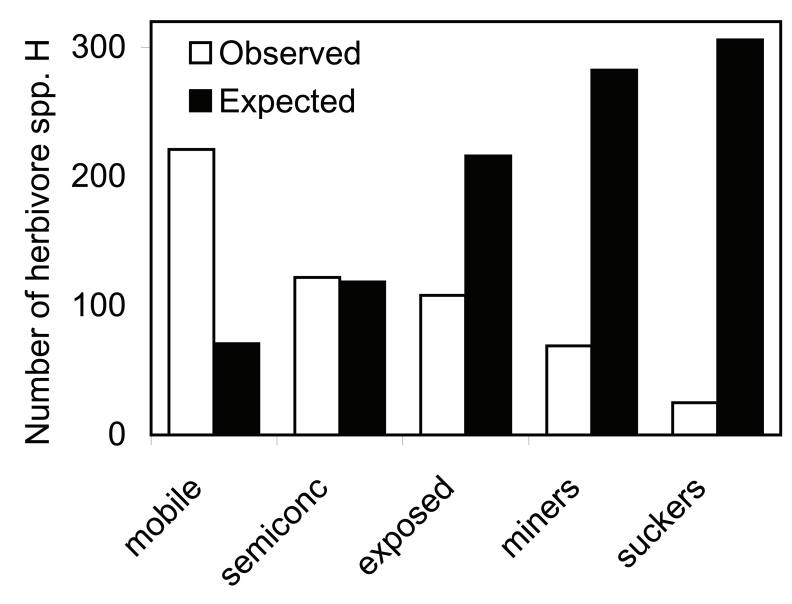

Figure 5: Observed and expected values of total herbivore species richness $H$ in five folivorous guilds. The expected values were estimated from equation (2), using empirical values of $\bar{p}_{i}$ and the constant value of $h_{j}=9.66$ for all guilds; $P=38$ studied plant species for all guilds. See table 1 for definitions of the variables.

function of land area (Rosenzweig 1995), so that the number of herbivore species should also increase as a power function of land area.

The accumulation of herbivore species with plant diversity can be described using the same function for both temperate and tropical regions, since the number of herbivore species per plant species and their host specificity do not differ between the two regions (Novotny et al. 2006; but see Dyer at al. 2007 for a different view). Tropical areas are particularly rich in plant species, which suggests higher relative diversity of specialized guilds such as miners and mesophyll suckers in the tropics than in the temperate zone. Unfortunately, the regional species pools of the most specialized guilds, such as miners and mesophyll suckers, are particularly poorly known taxonomically. For instance, our samples of Lepidoptera miners from New Guinea comprise almost entirely undescribed species, with the exception of a few widespread pest species (such as Neolithocolletis pentadesma feeding on Pterocarpus indicus; $\mathrm{Ku}-$ mata 1995). The extent of taxonomic knowledge is partly determined by body size (Gaston 1991), which tends to be small in the specialized guilds (Novotny and Basset 1999).

In conclusion, we have shown that host-specific guilds of insect herbivores form species-poor assemblages on individual plant species. This is contrary to the niche theory prediction that specialized use of resources will facilitate coexistence among species. Further indirect evidence that these guilds are not saturated with species comes from the guild composition, particularly the Poisson distribution of herbivore species richness among plant species, and the absence of a negative correlation between species richness in different guilds on the same plant. Species richness is more likely to be determined by the size of regional species pools for each guild and the way in which these pools are sampled by local communities.

\section{Acknowledgments}

We thank J. Auga, D. Bito, W. Boen, E. Brus, C. Dahl, K. Darrow, P. Drozd, L. Helgen, S. Ibalim, B. Isua, M. Janda, M. Keltim, J. Kua, R. Kutil, R. Lilip, M. Manumbor, M. Mogia, K. Molem, M. Rimandai, S. Sau, G. Setliff, G. Sosanika, E. Tamtiai, and D. Wal for technical assistance; V. O. Becker, J. Brown, L. Craven, K. Damas, D. R. Davis, J. D. Holloway, M. Horak, T. Kumata, K. Maes, J. Miller, E. G. Munroe, I. Ohshima, J. Rota, M. Shaffer, M. A. Solis, W. Takeuchi, K. Tuck, and T. Whitfeld for taxonomic assistance; P. Hebert for DNA bar codes; and T. Lewinsohn and two anonymous reviewers for comments on the manuscript. This work was supported by the National Science Foundation (DEB-9628840, -9707928, -0211591, and -0515678), the Grant Agencies of the Czech Republic (206/ 09/0115, 206/08/H044, and P505/10/0673), the Czech Academy of Sciences (AA600960712 and AV0Z50070508), the Czech Ministry of Education (LC06073, LH11008, ME9082, and MSM6007665801), the Darwin Initiative for the Survival of Species (UK), the Center for Tropical Forest Science/Smithsonian Institution Global Earth Observatory, and the David and Lucile Packard Fellowship in Science and Engineering (to G.D.W.). DNA bar coding was provided by the Biodiversity Institute of Ontario, with the support of Genome Canada under the iBOL project.

\section{Literature Cited}

Agrawal, A. A. 2007. Macroevolution of plant defence strategies. Trends in Ecology \& Evolution 22:103-109.

Andrew, N. R., and L. Hughes. 2005. Herbivore damage along a latitudinal gradient: relative impacts of different feeding guilds. Oikos 108:176-182.

Behmer, S. T., and A. Joern. 2008. Coexisting generalist herbivores occupy unique nutritional feeding niches. Proceedings of the National Academy of Sciences of the USA 105:1977-1982.

Caley, M. J., and D. Schluter. 1997. The relationship between local and regional diversity. Ecology 78:70-80.

Chase, J. M., and M. A. Leibold. 2003. Ecological niches: linking classical and contemporary approaches. University of Chicago Press, Chicago.

Claridge, M. F., and M. R. Wilson. 1981. Species richness of mesophyll-feeding leafhoppers and leafminers of trees in Britain. Acta Entomologica Fennica 38:19-20. 
Colwell, R. K. 2008. EstimateS: statistical estimation of species richness and shared species from samples. http://www.purl.oclc.org/ estimates. University of Connecticut, Storrs.

Condon, M. A., S. J. Scheffer, M. L. Lewis, and S. M. Swensen. 2008. Hidden Neotropical diversity: greater than the sum of its parts. Science 320:928-931.

Cornell, H. V., and D. M. Kahn. 1989. Guild structure in the British arboreal arthropods: is it stable and predictable? Journal of Animal Ecology 58:1003-1020.

Craft, K. J., S. U. Pauls, K. Darrow, S. E. Miller, P. N. D. Hebert, L. Helgen, V. Novotny, and G. D. Weiblen. 2010. Population genetic differentiation of New Guinea lowland rainforest Lepidoptera. Proceedings of the National Academy of Sciences of the USA 107: 5041-5046.

Dres, M., and J. Mallet. 2002. Host races in plant-feeding insects and their importance in sympatric speciation. Philosophical Transactions of the Royal Society B: Biological Sciences 357:471-492.

Dyer, L. A., M. S. Singer, J. T. Lill, J. O. Stireman, G. L. Gentry, R. J. Marquis, R. E. Ricklefs, et al. 2007. Host specificity of Lepidoptera in tropical and temperate forests. Nature 448:696-700.

Farrell, B. D. 1998. "Inordinate fondness" explained: why are there so many beetles? Science 281:555-559.

Fox, L. R., and P. A. Morrow. 1981. Specialization: species property or local phenomenon? Science 211:887-893.

Frenzel, M., and R. Brandl. 2000. Phytophagous insect assemblages and the regional species pool: patterns and asymmetries. Global Ecology and Biogeography 9:293-303.

Gaston, K. J. 1991. Body size and probability of description: the beetle fauna of Britain. Ecological Entomology 16:505-508.

Harrison, S., and H. Cornell. 2008. Toward a better understanding of the regional causes of local community richness. Ecology Letters 11:969-979.

Hespenheide, H. A. 1991. Bionomics of leaf-mining insects. Annual Reviews of Entomology 36:535-560.

Hoft, R. 1992. Plants of New Guinea and the Solomon Islands: dictionary of the genera and families of flowering plants and ferns. Handbook 13. Wau Ecology Institute, Wau.

Hulcr, J., S. E. Miller, G. P. Setliff, K. Darrow, N. D. Mueller, P. D. N. Hebert, and G. D. Weiblen. 2007a. DNA barcoding confirms polyphagy in a generalist moth, Homona mermerodes (Lepidoptera: Tortricidae). Molecular Ecology Notes 7:549-557.

Hulcr, J., M. Mogia, B. Isua, and V. Novotny. 2007b. Host specificity of ambrosia and bark beetles (Col., Curculionidae: Scolytinae and Platypodinae) in a New Guinea rainforest. Ecological Entomology 32:762-772.

Jaenike, J. 1990. Host specialization in phytophagous insects. Annual Reviews of Ecology and Systematics 21:243-273.

Janz, N., and S. Nylin. 2008. The oscillation hypothesis of host plantrange and speciation. Pages 203-215 in K. J. Tilmon, ed. Specialization, speciation, and radiation: the evolutionary biology of herbivorous insects. University of California Press, Berkeley.

Joshi, A., and J. N. Thompson. 1995. Trade-offs and the evolution of host specialization. Evolutionary Ecology 9:82-92.

Kelly, C. K., and T. R. E. Southwood. 1999. Species richness and resource availability: a phylogenetic analysis of insects associated with trees. Proceeding of the National Academy of Sciences of the USA 96:8013-8016.

Kishimoto-Yamada, K., and T. Itioka. 2008. Survival of flower-visiting Chrysomelids during non general-flowering periods in Bornean dipterocarp forests. Biotropica 40:600-606.
Kumata, T. 1995. Some species of the subfamily Lithocolletinae (Gracillariidae, Lepidoptera) collected in the Philippines. Insecta Matsumurana, New Series 52:105-131.

Lawton, J. H., T. M. Lewinsohn, and S. G. Compton. 1993. Patterns of diversity for the insect herbivores on bracken. Pages 178-184 in R. E. Ricklefs and D. Schluter, eds. Species diversity in ecological communities: historical and geographical perspectives. University of Chicago Press, Chicago.

Leibold, M. A., M. Holyoak, N. Mouquet, P. Amarasekare, J. M. Chase, M. F. Hoopes, R. D. Holt, et al. 2004. The metacommunity concept: a framework for multi-scale community ecology. Ecology Letters 7:601-613.

Leigh, E. G. J., P. Davidar, C. W. Dick, J.-P. Puyravaud, J. Terborgh, H. ter Steege, and S. J. Wright. 2004. Why do some tropical forests have so many species of trees? Biotropica 36:447-473.

Lewinsohn, T. M., and T. Roslin. 2008. Four ways towards tropical herbivore megadiversity. Ecology Letters 11:398-416.

Martins, E. P. 2003. COMPARE 4.6. Computer programs for the statistical analysis of comparative data. Department of Biology, Indiana University, Bloomington.

Miller, S. E., V. Novotny, and Y. Basset. 2003. Studies on New Guinea moths. I. Introduction (Lepidoptera). Proceedings of the Entomological Society of Washington 105:1034-1042.

Mitter, C., B. Farrell, and B. Wiegmann. 1988. The phylogenetic study of adaptive zones: has phytophagy promoted insect diversification? American Naturalist 132:107-128.

Nosil, P. 2002. Transition rates between specialization and generalization in phytophagous insects. Evolution 56:1701-1706.

Nosil, P., B. J. Crespi, and C. P. Sandoval. 2002. Host-plant adaptation drives the parallel evolution of reproductive isolation. Nature 417: 440-443.

Novotny, V., and Y. Basset. 1999. Body size and host plant specialisation: a relationship from a community of herbivorous insects from New Guinea. Journal of Tropical Ecology 15:315-328.

2000. Rare species in communities of tropical insect herbivores: pondering the mystery of singletons. Oikos 89:564-572.

- 2005. Host specificity of insect herbivores in tropical forests. Proceedings of the Royal Society B: Biological Sciences 272:10831090.

Novotny, V., and O. Missa. 2000. Local and regional species richness in the tropics: sap-sucking insects (Auchenorrhyncha, Hemiptera) from a lowland rain forest site and the island of New Guinea. Ecological Entomology 25:445-451.

Novotny, V., and M. R. Wilson. 1997. Why are there no small species among xylem-sucking insects? Evolutionary Ecology 11:419-437.

Novotny, V., Y. Basset, S. E. Miller, P. Drozd, and L. Cizek. 2002. Host specialisation of leaf chewing insects in a New Guinea rainforest. Journal of Animal Ecology 71:400-412.

Novotny, V., A. R. Clarke, R. A. I. Drew, S. Balagawi, and B. Clifford. 2005. Host specialization and species richness of fruit flies (Diptera: Tephritidae) in a New Guinea rain forest. Journal of Tropical Ecology 21:67-77.

Novotny, V., P. Drozd, S. E. Miller, M. Kulfan, M. Janda, Y. Basset, and G. D. Weiblen. 2006. Why are there so many species of herbivorous insects in tropical rainforests? Science 313:1115-1118.

Novotny, V., S. E. Miller, J. Hulcr, R. A. I. Drew, Y. Basset, M. Janda, G. P. Setliff, et al. 2007. Low beta diversity of herbivorous insects in tropical forests. Nature 448:692-695.

Novotny, V., S. E. Miller, L. Baje, S. Balagawi, Y. Basset, L. Cizek, K. J. Craft, et al. 2010. Guild-specific patterns of species richness and 
host specialization in plant-herbivore food webs from a tropical forest. Journal of Animal Ecology 79:1193-1203.

Nylin, S., and N. Janz. 2009. Butterfly host plant range: an example of plasticity as a promoter of speciation? Evolutionary Ecology 23: 137-146.

Nyman, T., B. D. Farrell, A. G. Zinovjev, and V. Vikberg. 2006. Larval habits, host-plant associations, and speciation in nematine sawflies (Hymenoptera: Tenthredinae). Evolution 60:1622-1637

Pokon, R., V. Novotny, and G. A. Samuelson. 2005. Host specialization and species richness of root feeding chrysomelid larvae (Chrysomelidae, Coleoptera) in a New Guinea rainforest. Journal of Tropical Ecology 21:595-604.
Ricklefs, R. E. 2004. A comprehensive framework for global patterns in biodiversity. Ecology Letters 7:1-15.

Rosenzweig, M. L. 1995. Species diversity in space and time. Cambridge University Press, Cambridge.

Shmida, A., and M. V. Wilson. 1985. Biological determinants of species diversity. Journal of Biogeography 12:1-20.

Simberloff, D., and T. Dayan. 1991. The guild concept and the structure of ecological communities. Annual Review of Ecology and Systematics 22:115-143.

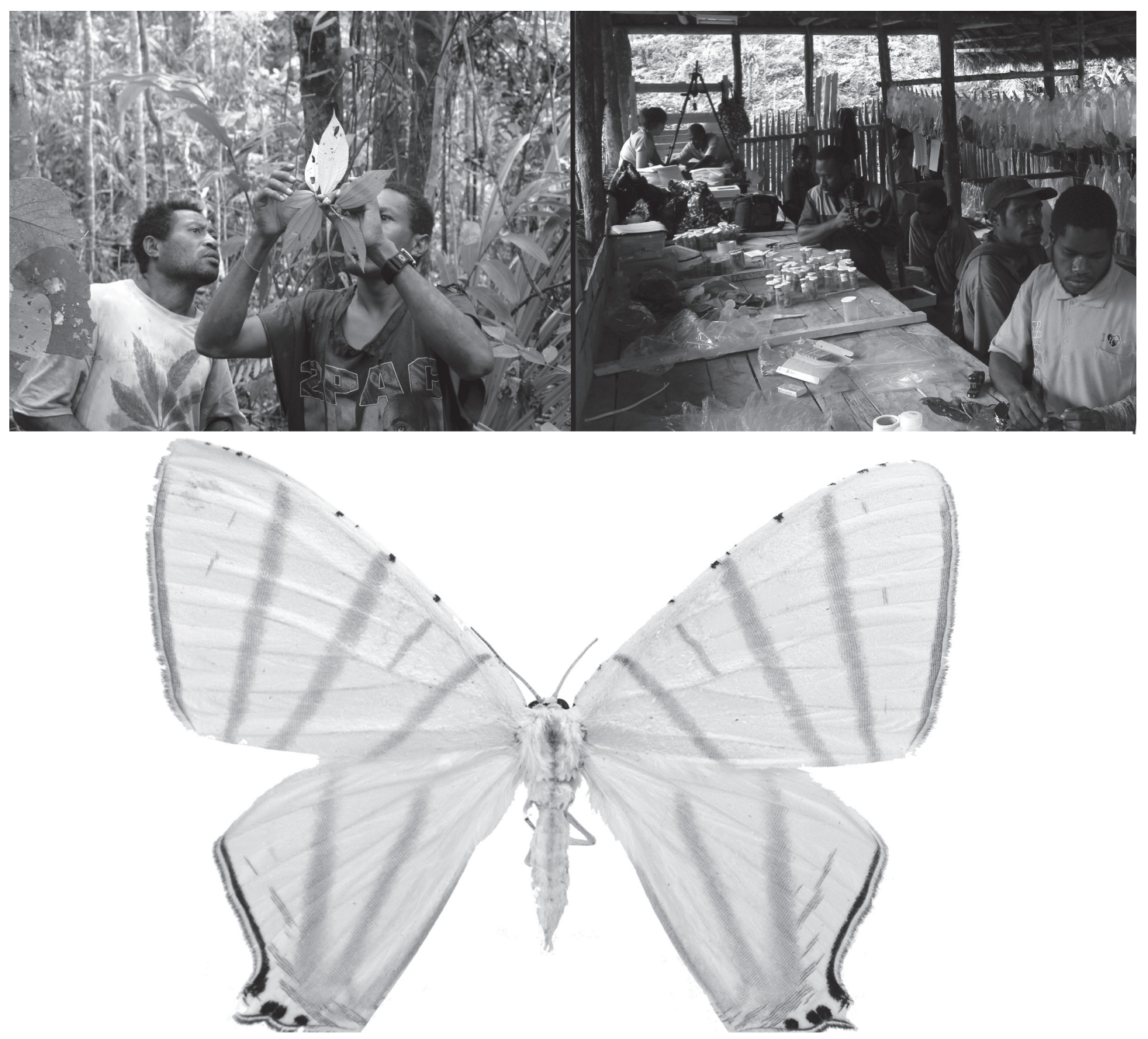

Top left and right, sampling and rearing insects in a rainforest laboratory. Photographs by Vojtech Novotny. Bottom, the moth Cyphura swinhoei Joicey \& Talbot (Uraniidae) feeds on Endospermum labios, an ant tree in the New Guinea rainforest. Photograph by Lauren Helgen. 